\title{
SPECTROMÉTRIE PAR LASERS DE LONGUEUR D'ONDE VARIABLE
}

\author{
J. M. BESSON \\ Laboratoire de Physique de l'Ecole Normale Supérieure, Paris Ve
}

\begin{abstract}
Résumé. - Il est possible de faire varier la longueur d'onde d'émission de lasers semiconducteurs par 1'application de pressions hydrostatiques.

Cette variation peut s'étendre sur plusieurs octaves du spectre et couvrir les longueurs d'onde de 1 à 20 microns, au moins, suivant le semiconducteur utilisé.

L'application de cette technique à la spectrométrie infrarouge permettra de tirer parti de la très grande brillance de ces sources, de leur pureté spectrale et de leur grande souplesse de modulation en haute fréquence.
\end{abstract}

\begin{abstract}
It is possible to vary continuously the emission wavelength of semiconductor lasers by hydrostatic pressure. This variation can extend over several spectral octaves and cover the 1-20 micron range at least, depending on the material.

The use of this technique for infrared spectrometry will provide sources of high brightness, good spectral purity and attractive high-frequency modulation capabilities.
\end{abstract}

Certains lasers semiconducteurs peuvent émettre à longueur d'onde variable, sous l'action de contraintes hydrostatiques. Cette propriété jointe à la grande brillance de ces dispositifs peut être mise à profit pour la réalisation de sources monochromatiques variables dans le domaine des longueurs d'onde infrarouge.

Les cristaux semiconducteurs utilisés pour la fabrication de structures laser appartiennent, à l'exception des sels de plomb, à la famille cristalline diamantblende. Ces composés III-V présentent qualitativement la même structure de bandes. Leurs différences proviennent de la position relative des extremums de bandes. En particulier, si la transition de bande interdite est directe et permise, il peut apparaître des phénomènes d'amplification radiative à une énergie correspondant à la largeur de la bande interdite.

La structure de bande des semiconducteurs peut se modifier sous l'action de contraintes hydrostatiques qui permettent de faire varier de façon considérable la position relative des extremums de bandes. Cette variation est prévisible grâce à la règle suivante établie par W. Paul et ses collaborateurs : " Le coefficient de pression de la différence d'énergie de deux niveaux électroniques de symétrie donnée est le même pour tous les membres d'une même famille cristalline. ) Dans la famille diamant-blende, la transition de bande interdite directe $\Gamma_{15}^{\mathrm{v}} \rightarrow \Gamma_{1}^{\mathrm{c}}$ pour les composés III-V aura un coefficient de pression [1] $\mathrm{de}+12 \pm 2 \times 10^{-6}$ eV.bar ${ }^{-1}$.
Les sels de plomb, $\mathrm{PbS}, \mathrm{PbSe}$ et $\mathrm{PbTe}$, appartiennent à la famille de $\mathrm{NaCl}$ et ont des extremums en bord de zone de Brillouin, au point $\mathrm{L}$ [111]. Leurs bandes interdites ont des coefficients de pression de $-8 \times$ $\times 10^{-6} \mathrm{eV}^{\mathrm{bar}}{ }^{-1}$.

L'effet des contraintes uniaxes sur les structures de bandes est, bien entendu, plus complexe, puisque, suivant leur direction d'application et la symétrie des niveaux étudiés, elles peuvent, soit faire varier l'énergie de ces niveaux, soit, en plus, enlever les dégénérescences et les séparer en plusieurs sous-niveaux. Ce type de contraintes, de mise en cuvre relativement aisée, ne semble pas, pour l'instant, avoir un domaine d'application aussi étendu que les contraintes hydrostatiques.

Il est possible d'obtenir dans de nombreux cristaux semiconducteurs des régimes d'amplification radiative et même d'oscillation de cavités à des longueurs d'onde très proches de celles correspondant à l'énergie de bande interdite. L'effet laser a été observé sur de nombreux composés III-V comme les antimoniures, phosphures et arséniures d'indium et de gallium, et aussi sur les sulfures et séléniures de cadmium, les tellures mixtes de mercure et de cadmium, et les sulfures, séléniures et tellurures de plomb. Les trois méthodes usuelles d'excitation leur sont applicables, c'est-à-dire le bombardement électronique, le pompage optique et l'injection de porteurs. Seules les deux dernières sont applicables à des études sous pression hydrostatique. 
Quelle que soit la nature du laser étudié, il existe un certain nombre de paramètres que la pression fera varier de façon analogue. Ce sont, entre autres :

- la longueur d'onde d'émission,

- les dimensions de la cavité,

- l'indice du milieu amplificateur.

La longueur d'onde d'émission stimulée est, pour la plus grande partie, déterminée par la longueur d'onde de l'émission spontanée correspondante. Elle variera donc avec sensiblement le même coefficient de pression que la bande interdite. Cette variation se fera donc vers les courtes longueurs d'onde pour les semiconducteurs à coefficient de pression positif comme GaAs, et vers les grandes longueurs d'onde pour les sels de plomb.

L'indice variera de façon sensible pour les corps à faible bande interdite, puisqu'il a une singularité [2] pour $E_{g}=0$. Il détermine, entre autres, le gain du milieu amplificateur et la réflectivité aux interfaces de la cavité.

Les premières études sur la variation avec la pression de la longueur d'onde d'émission et des paramètres radiatifs de diodes ont été faites sur des jonctions de GaAs [3]. Le coefficient de pression étant d'environ
$+10^{-5}$ eV $\cdot$ bar $^{-1}$ et la bande interdite de $1,5 \mathrm{eV}$ à $77 \mathrm{oK}$, la variation de longueur d'onde entre 1 et 8000 bars était, dans ces mesures, de l'ordre de $6 \%$.

Pour les sels de plomb, et en particulier PbSe, la variation est beaucoup plus importante, puisque leur coefficient de pression est négatif et de l'ordre de $-8 \times 10^{-6} \mathrm{eV} \cdot \mathrm{bar}^{-1}$ pour une bande interdite de $175 \mathrm{meV}$. On peut donc s'attendre, entre autres, à annuler la bande interdite à des pressions voisines de 20 kilobars.

La variation corrélative de la longueur d'onde d'émission de diodes laser de PbSe a été observée récemment de 7,5 à $11 \mu$ [4], puis jusqu'à $22 \mu$ [5] avec des pressions de l'ordre de 14 kilobars. Le dispositif de pression était composé d'un générateur classique à trois étages de compression d'hélium, et d'une chambre de pression contenant la diode, reliée au générateur par un tuyau souple, et plongée dans un cryostat à azote liquide.

La variation de l'énergie des photons émis en fonction de la pression donne une loi presque linéaire dont la pente est $-8,5 \times 10^{-6} \mathrm{eV} \cdot$ bar $^{-1}$ à 1 bar et

$$
-7,5 \times 10^{6} \mathrm{eV} \cdot \mathrm{bar}^{-1}
$$

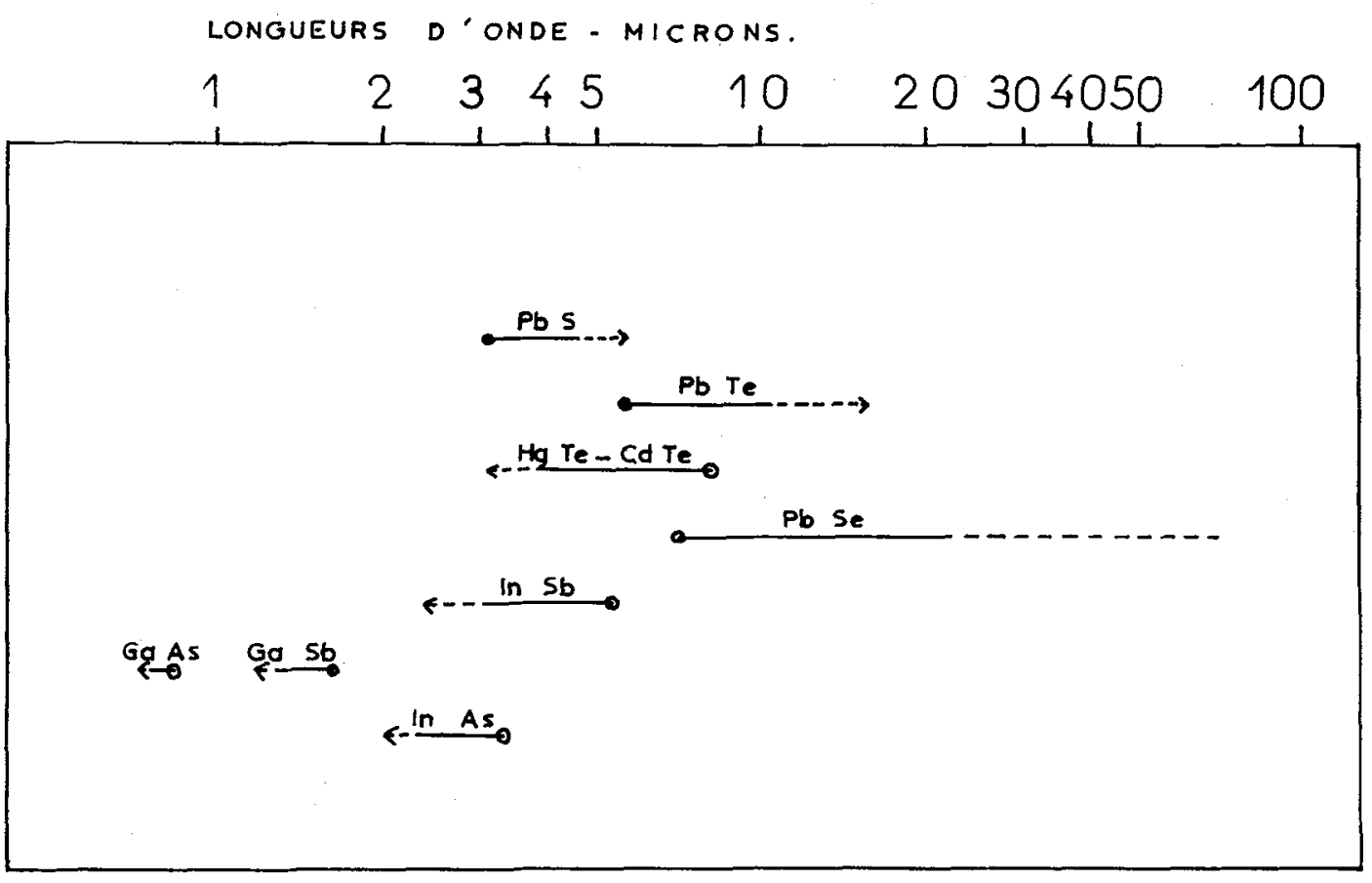

FIG. 1. - Longueur d'onde approximative de l'émission de lasers semi-conducteurs soumis à des pressions hydrostatiques à $77^{\circ} \mathrm{K}$.

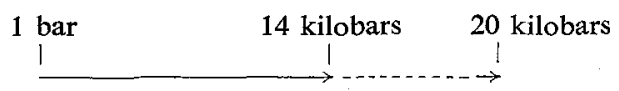

Ces évaluations sont, soit déduites de mesures directes, soit calculées d'après les coefficients de pression expérimentaux des divers cristaux. 
à 14000 bars. Le régime d'émission stimulée et les modes de la cavité se conservent tout le long du domaine exploré. Cette pression-limite de 14 kilobars est imposée par la pression de solidification de l'hélium à $77^{\circ} \mathrm{K}$. Pour aller plus loin, il faudra utiliser un dispositif de compression directe d'hélium solide, donc un milieu quasi-hydrostatique.

Ces dispositifs cohérents à longueur d'onde variable peuvent s'appliquer aux techniques de la spectroscopie infrarouge qui manque actuellement de sources brillantes. Les détecteurs refroidis approchent maintenant la limite du bruit de fond thermique à $300^{\circ} \mathrm{K}$ et la qualité optique des spectromètres dans l'infrarouge moyen et lointain est plus que suffisante. C'est donc surtout la faible brillance des sources à incandescence classiques qui limite la résolution effective qu'on peut atteindre dans l'infrarouge. D'autre part, les dispositifs d'émission cohérente sont rarement utilisables audessous du millimètre de longueur d'onde. Les dispositifs utilisant l'effet Smith-Purcell sont de très faible brillance. Les lasers à gaz, eux, n'ont pas de possibilité d'accord. Quant aux sources à battements de lasers ou à génération d'hypersons, elles en sont au stade théorique ou aux très faibles puissances, de même que celle utilisant l'effet Cerenkov cohérent.

Par contre, les lasers semiconducteurs ont les caractéristiques suivantes :

- Faibles dimensions de l'ordre de 100 à $1000 \mu^{2}$ pour la partie active.

- Directivité de l'émission.

- Finesse des raies pouvant atteindre $10^{9}$ [6].

- Puissances comprises entre $10^{-6}$ et $10^{1}$ watts selon le matériau considéré. Les bons lasers à GaAs donnent des flux supérieurs à $10^{5} \mathrm{~W} / \mathrm{cm}^{2}$ sans focalisation. Ces résultats ne valent que pour les lasers à GaAs dont la technologie est la mieux étudiée. Cependant, même si l'on devait en rester pour les autres matériaux laser aux puissances actuelles, ceux-ci ont tout de même des brillances monochromatiques supérieures de plusieurs ordres de grandeur à celles des sources à incandescence.

Les lasers semiconducteurs peuvent être utilisés à peu près sur tout le spectre infrarouge de 2 à $22 \mu$, comme le montre la figure, et probablement plus loin. Leur utilisation systématique en spectrométrie et en irradiation infrarouge est principalement une question de technologie pour la stabilisation et le contrôle de la pression et du courant d'injection. Elle permettra de tirer parti de la finesse des raies d'émission laser et de leur souplesse de modulation en haute fréquence. Enfin, elle ne manquera pas de bénéficier directement des progrès rapides de la technologie des lasers semiconducteurs.

\section{Bibliographie}

[1] ZaLLEN (R.), Thèse, Harvard University, 1964.

[2] Stern (F.), Phys. Rev., 1964, 133, A 1653.

[3] Feinleib (J.), Groves (S.), Paul (W.), Zallen (R.), Phys. Rev., 1963, 131, 2070.

[4] Besson (J. M.), Butler (J. F.), Calawa (A. R.), Paul (W.), ReDIKER (R. H.), Appl. Phys. Let., 1965, $7,206$.

[5] Besson (J. M.), Calawa (A. R.), Paul (W.), à paraître.

[6] Ahearn (W. E.), Crowe (J. W.), "Linewidth measurements of CW gallium arsenide lasers at $77^{\circ} \mathrm{K}$ 》, International Quantum Electronics Conference, 1966. 